\title{
Prospects for Cross-correlations of UHECR Events with Astrophysical Sources with Upcoming Space-based Experiments
}

\section{Tonia M. Venters ${ }^{a, *}$ and Andrés Romero-Wolf ${ }^{b}$ on behalf of the POEMMA and ZAP Collaborations}

(a complete list of authors can be found at the end of the proceedings)

\author{
${ }^{a}$ NASA Goddard Space Flight Center, \\ Astrophysics Science Division, Greenbelt, Maryland, USA \\ ${ }^{b}$ Jet Propulsion Laboratory, \\ California Institute of Technology, Pasadena, California, USA \\ E-mail: tonia.m.venters@nasa.gov, andrew.romero-wolf@jpl.nasa.gov
}

\begin{abstract}
Ultra-high energy cosmic rays (UHECRs) are the messengers of the most extreme physics in the cosmos; however, efforts to identify their origins have thus far been thwarted by the fact that they don't point back to their sources. Using statistical studies cross-correlating UHECR arrival directions with astrophysical catalogs, the ground-based Pierre Auger Observatory has reported hints of a correlation with nearby starburst galaxies, as well as lower-significance correlations with other classes of astrophysical sources. Space-based UHECR experiments, such as POEMMA and ZAP, will monitor large interaction volumes on the Earth or the Moon. Within a few years of mission operation time, both missions will achieve unprecedented exposures at energies above $40 \mathrm{EeV}$ across the entire sky. In this contribution, we present studies of the cross-correlation between UHECR event arrival directions and astrophysical catalogs as motivated by expectations for the detector performance for POEMMA and ZAP. We find that both POEMMA and ZAP will achieve $5 \sigma$ discovery reach for many plausible astrophysical scenarios.
\end{abstract}

$37^{\text {th }}$ International Cosmic Ray Conference (ICRC 2021)

July 12 th - 23rd, 2021

Online - Berlin, Germany

\footnotetext{
${ }^{*}$ Presenter
} 


\section{Introduction}

Even after more than 80 years since the first detailed indirect investigations of high-energy cosmic rays via extensive air showers (EASs; [1]), the origins of these energetic particles, particularly those with ultra-high energies (UHEs; $E \gtrsim 10^{18} \mathrm{eV}$ ), remain a mystery. Deflections by equally mystifying magnetic fields both inside and outside of the Galaxy hinder efforts to directly track UHECRs back to their sources. Weaker deflections at the highest energies would, in principle, allow UHECRs to arrive closer to the positions of their sources, but the exact energy threshold beyond which this occurs has yet to be determined. At similar energies, UHECRs interact with cosmological photon backgrounds (via, e.g., the GZK effect, $[2,3]$ ) resulting in a horizon distance ( $\sim 1 \mathrm{Gpc}$ at $E \sim 10^{19} \mathrm{eV}$ and dropping to $\sim$ few hundreds of Mpc beyond $5 \times 10^{19} \mathrm{eV}$; [see e.g., 4]) within which the highest energy CRs must originate. Out to this distance, the local matter distribution, including nearby UHECR sources, is anisotropic; hence, the UHECR sky distribution is likewise expected to be anisotropic and may even exhibit hot spots around powerful, nearby sources. Searches for anisotropy in the distribution of UHECR arrival directions have provided the first high-significance detection (significance $\sim 6 \sigma$ ) by the Pierre Auger Observatory of a dipolar modulation (amplitude $d_{\perp} \sim 6 \%$ pointing far away from the Galactic Center) in events with energies above $8 \mathrm{EeV}[5,6]$, confirming the extragalactic origins of UHECRs. Both Auger and the Telescope Array (TA) have reported hints of intermediate-scale $\left(\sim 20^{\circ}\right)$ anisotropy in the form of hot spots in the sky distributions of UHECR events with energies above $41 \mathrm{EeV}$ (post-trial p-value or 1.4\%) and $57 \mathrm{EeV}$ (significance $\sim 3.2 \sigma$ ), respectively [7, 8].

Another approach to probing anisotropy on intermediate scales is to search for cross-correlations between UHECR arrival directions and astrophysical catalogs. Such studies compare the spatial clustering of UHECR events with those of candidate astrophysical sources, possibly providing clues about UHECR sources and their characteristics, as well as the amount of deflection due to intervening magnetic fields [9]. Statistical cross-correlation studies performed by Auger have provided intriguing hints of a correlation with nearby astrophysical sources, with the strongest signal arising from starburst galaxies (significance $\sim 4.0 \sigma ;[7]$ ). With a significant increase in exposure above $\sim 40 \mathrm{EeV}$, these hints could become more definite signals [10,11].

A key science driver for any future UHECR experiment is achieving the performance required in order to firmly connect UHECRs with their sources via the aforementioned anisotropy searches. In this work, we assess the science reach for cross-correlation studies of UHECR anisotropy with

\begin{tabular}{|c|c|c|}
\hline \hline Mission Specification & $\begin{array}{c}\text { POEMMA } \\
\text { (Stereo Mode) }\end{array}$ & ZAP \\
\hline \hline Energy Resolution & $\begin{array}{c}\leq 18 \% \text { above } \\
50 \mathrm{EeV}\end{array}$ & $<30 \%$ \\
\hline Angular Resolution & $\begin{array}{c}<1.5^{\circ} \mathrm{above} \\
40 \mathrm{EeV}\end{array}$ & $1^{\circ}-4^{\circ}$ \\
\hline$N_{\mathrm{ev}}$ with $E \gtrsim 40 \mathrm{EeV}$ & 1400 & 2000 \\
\hline Operation Timescale & 5 years & 2 years \\
\hline \hline
\end{tabular}

Table 1: Select list of mission specifications for POEMMA and ZAP. 

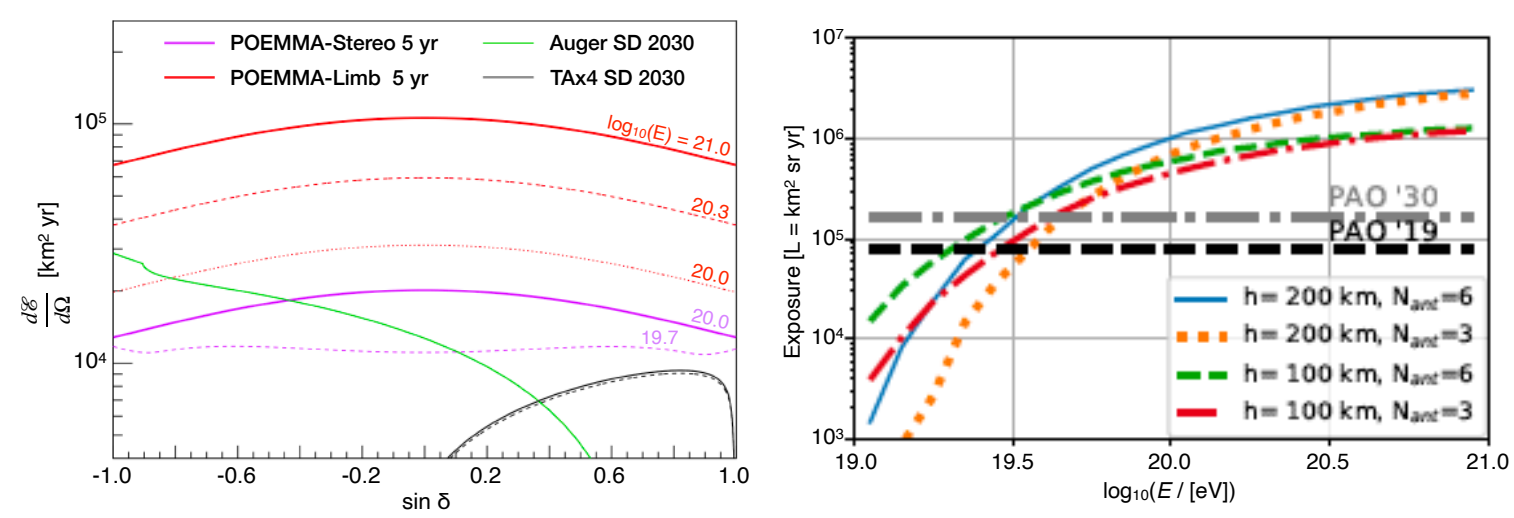

Figure 1: Left: Projected differential exposure for five years of POEMMA as a function of declination at various energies for two operational modes. Figure reproduced from [14]. Right: Exposure projections for two years of ZAP as a function of the logarithm of the energy for different orbit altitudes and numbers of antennae. The current (black dashed) and 2030-projected (grey dot-dashed) exposures for the Pierre Auger Observatory are provided for comparison. Figure reproduced from [13].

two future experiments, the Probe of Extreme MultiMessenger Astrophysics (POEMMA) [12] and the Zettavolt Askaryan Polarimeter (ZAP) [13]. Both experiments are space-based missions that will monitor larger interaction volumes in order to achieve large increases in exposure relative to current experiments and planned enhancements as seen in Fig. 1. Both missions will also feature full-sky coverage, providing the capability to observe the entire UHECR source distribution over the celestial sphere, and eliminating the need for cross calibration between different experiments with only partial sky coverage.

POEMMA will orbit the Earth in search of fluorescence signals from EASs of UHECRs and neutrinos above $20 \mathrm{EeV}$ and optical Cherenkov signals from upward-going EASs of tau neutrinos above $10 \mathrm{PeV} .{ }^{1}$ The observatory design consists of two identical telescopes on board individual satellites orbiting in tandem with a separation of $\sim 300 \mathrm{~km}$ at an altitude of $525 \mathrm{~km}$. POEMMA will operate in two science observation modes: POEMMA-Stereo mode in which the telescopes are pointed close to nadir to monitor overlapping atmospheric volumes, and POEMMA-Limb in which the telescopes are tilted $\sim 45^{\circ}$ from nadir to monitor even larger atmospheric volumes below the Earth's limb. The POEMMA-Stereo mode is optimized for stereo fluorescence observations of UHECR EASs and enables excellent angular, energy, and composition resolution. While the POEMMA-Limb mode is optimized for optical Cherenkov observations, UHECR fluorescence observations will continue, albeit with an enhanced acceptance and less precise reconstruction capabilities [14]. In this work, we focus on the performance capabilities enabled by POEMMAStereo mode (see Table 1).

ZAP will orbit the Moon in search of Askaryan radio signals [15, 16] from UHECR showers developing in the lunar regolith. The observatory design consists of an array of dipole antennas with baseline separations of $\sim 5.7 \mathrm{~m}$ in order to localize the direction of the radio impulse to within an uncertainty of $\sim 3^{\circ}$. The radio impulse is emitted over multiple frequencies with a beam pattern that varies from dipole-like at lower frequencies $(\lesssim 100 \mathrm{MHz})$ and transitions to cone-shaped at higher frequencies. ZAP will target the lower frequencies $(30-300 \mathrm{MHz})$ in order to take advantage

\footnotetext{
${ }^{1}$ The capabilities of POEMMA for detecting neutrinos are discussed in other proceedings.
} 

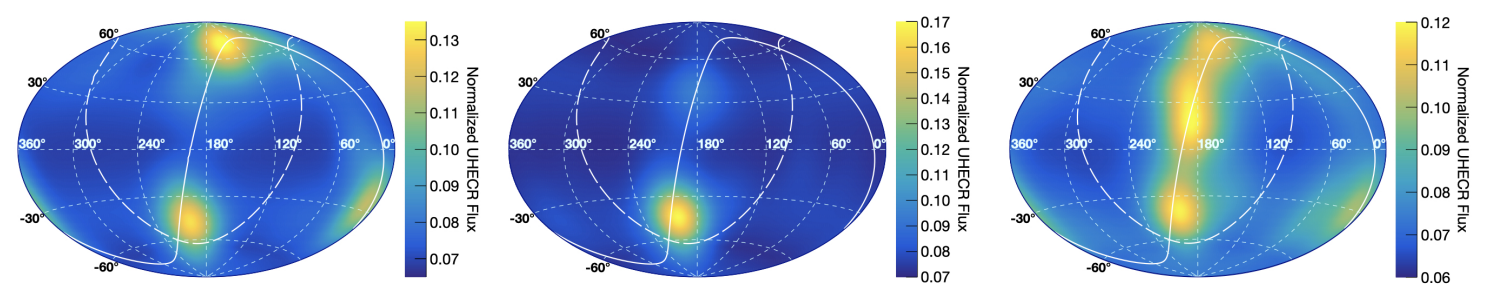

Figure 2: Sky plots of the normalized UHECR flux in equatorial coordinates for astrophysical scenarios with best-fit parameters reported by Auger [17]. The dashed white line is the Galactic Plane. The solid white line is the Supergalactic Plane. Left: Starburst galaxies with $f_{\text {sig }}=11 \%$ and $\Theta=15^{\circ}$. Middle: Swift-BAT AGNs [18] with $f_{\text {sig }}=8 \%$ and $\Theta=15^{\circ}$. Right: $2 M R S$ galaxies [19] with $f_{\text {sig }}=19 \%$ and $\Theta=15^{\circ}$. Figures reproduced from [12].

of the wider beam pattern to allow for UHECR detection in a wider range of viewing angles, increasing event acceptance.

\section{Anisotropy Searches by Cross-Correlating Arrival Directions of UHECR Events with Astrophysical Catalogs}

In this work, we follow the Bayesian approach to searching for cross correlations between UHECR arrival directions and astrophysical catalogs [20-22]. Astrophysical hypotheses are expressed as sky maps consisting of either a purely isotropic distribution for UHECR events or a mixed distribution consisting of an isotropic component and an anisotropic component arising from astrophysical sources:

$$
\mathcal{F}_{\text {sky }}(\hat{n})=\frac{w(\hat{n})}{C}\left[\left(1-f_{\text {sig }}\right) \frac{1}{4 \pi}+f_{\text {sig }} \mathcal{F}_{\text {src }}(\hat{n})\right],
$$

where $\mathcal{F}_{\text {sky }}(\hat{n})$ is the normalized overall UHECR flux sky map (including both isotropic and anisotropic components), $\hat{n}$ is the unit vector for a given location on the sky, $w(\hat{n})$ is the experiment's exposure in the direction of $\hat{n}$ (see Fig. 1), $f_{\text {sig }}$ is the signal fraction (the fraction of UHECRs originating from the sources), $\mathcal{F}_{\text {src }}$ is the normalized source sky map for the flux of UHECRs originating from the sources, and $C$ is a normalization factor to ensure that $\int \mathcal{F}_{\text {sky }}(\hat{n}) d \Omega=1$.

We construct $\mathcal{F}_{\text {src }}$ from a flux-limited astrophysical catalog, weighting each source by its electromagnetic flux (assuming that the UHECR flux is proportional to the electromagnetic flux) and applying an attenuation factor that accounts for UHECR energy losses through propagation over the distance to the source. Each source is also weighted by a von Mises-Fisher distribution ${ }^{2}$ with angular spread $\Theta$. For the source attenuation factors, we follow the data-driven approach of [23] that reproduces Auger composition and spectrum measurements [24]. In this work, we assume Scenario A of [23] in which the air-shower data are modeled using the EPOS-LHC interaction model and an at-source injection spectrum that is a power law with a hard index $(\gamma=1)$ and a rigidity-dependent cutoff.

For this study, we use the same astrophysical catalogs as in [17, 23], which include a catalog of starburst galaxies selected based on their continuum emission at $1.4 \mathrm{GHz}$, a catalog of radio-loud and radio-quiet AGNs included in the 70 Month Swift-BAT All-sky Hard X-ray Survey [18], and a

\footnotetext{
${ }^{2}$ The equivalent of a Gaussian distribution on the surface of a sphere. For the 2 -sphere, it is given by $\mathcal{G}(\hat{n}, \hat{s} ; \kappa)=$ $\kappa \exp (\hat{n} \cdot \hat{s}) /(4 \pi \sinh \kappa)$, where $\kappa=\Theta^{-2}$ is the concentration parameter.
} 
catalog of galaxies at distances greater than $1 \mathrm{Mpc}$ from the 2MASS Redshift Survey (2MRS) of nearby galaxies [19].

Fig. 2 provides example normalized UHECR flux sky maps constructed using Eqn. 1 and the three astrophysical catalogs. The parameters for the maps in Fig. 2 are selected to coincide with the best-fit parameters reported by Auger [17] for the given astrophysical catalog.

The cross-correlation test takes the form of a Bayesian hypothesis test in which alternative astrophysical hypotheses are tested against a null hypothesis (isotropy, in this case). For a given UHECR dataset, an alternative astrophysical hypothesis is assigned a test statistic (TS) that is defined by the ratio of the likelihood that the dataset is drawn from the sky map corresponding to parameter set $\left(f_{\text {sig }}, \Theta\right)$ to the likelihood that it is drawn from isotropy:

$$
\mathrm{TS}=2 \ln \left(\frac{L\left(\mathcal{F}_{\text {sky }}\right)}{L\left(\mathcal{F}_{\text {iso }}\right)}\right)
$$

where $\mathcal{F}_{\text {iso }}=w(\hat{n}) / 4 \pi$. The likelihood is given by

$$
L(\mathcal{F})=\frac{1}{\mathcal{N}} \prod_{i} \mathcal{F}\left(\hat{n}_{i}\right)
$$

where $h a t n_{i}$ is the unit vector for the arrival direction of the $i^{\text {th }}$ UHECR event and $\mathcal{N}$ is a normalization factor. By varying $f_{\text {sig }}$ and $\Theta$, the maximum TS value can be obtained (see example in Fig. 3). The significance is calculated using a $\chi^{2}$ distribution with two degrees of freedom [25]. In cross-correlation searches with real UHECR datasets, a scan over the energy threshold may be performed to obtain a global maximum, though at the expense of penalization [e.g., 26].

\section{Prospects for Detecting Cross-correlations between UHECR Events and Astrophysical Sources with POEMMA and ZAP}

In this work, we perform the statistical analysis outlined in Section 2 (see also [14]) on simulated UHECR data sets for POEMMA and ZAP. In so doing, we seek to determine: (1) the on-average significance of the cross correlation in a data set with a pre-determined number of events in a given astrophysical scenario, and (2) the number of events needed to guarantee a $5 \sigma$ detection of the cross correlation in a given astrophysical scenario.

Simulated UHECR data sets represent particular scenarios and are parameterized ${ }^{3}$ by the total number of events, $N_{\mathrm{ev}}$, the fraction $f_{\mathrm{an}}^{*}$ of events that deviate from isotropy, and the spread $\Theta^{*}$ of the UHECR flux around the sources. In scenarios in which $f_{\mathrm{an}}^{*}>0$, we randomly generate the anisotropic subset of UHECR events from source sky maps constructed from the astrophysical catalogs (see Section 2) with individual source fluxes smoothed by $\Theta^{*}$. The rest of the UHECR events $\left(\left(1-f_{\mathrm{an}}^{*}\right) \times N_{\mathrm{ev}}\right.$ are drawn from an isotropic sky maps. All sky maps used to construct the mock data

\footnotetext{
${ }^{3}$ Note that we use $\left(^{*}\right)$ to distinguish parameters used to generate the mock data sets from the search parameters of the statistical analysis.
} 


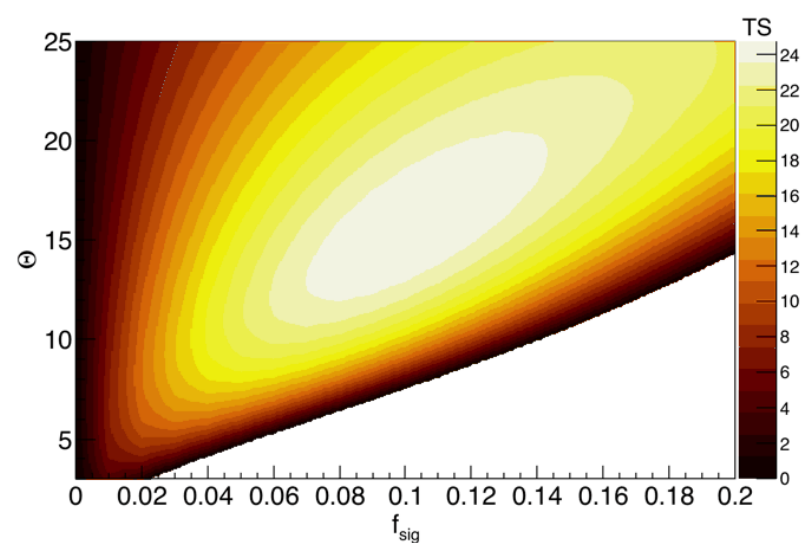

Figure 3: Example TS profile from the likelihood parameter study of a mock UHECR dataset (see Section 3). In the scenario pictured here, the source sky map is the SBG map, and the parameters for the mock dataset are $N_{\mathrm{ev}}=1400, f_{\mathrm{an}}^{*}=11 \%$, and $\Theta^{*}=15^{\circ}$. Figure reproduced from [14].

\begin{tabular}{|c|ccc|}
\hline \hline Catalog & $f_{\text {sig }}$ & TS & $\sigma$ \\
\hline & $5 \%$ & 6.2 & 2.0 \\
SBG & $10 \%$ & 24.7 & 4.6 \\
& $15 \%$ & 54.2 & 7.1 \\
& $20 \%$ & 92.9 & 9.4 \\
\hline \multirow{3}{*}{ 2MRS } & $5 \%$ & 2.4 & 1.0 \\
& $10 \%$ & 8.7 & 2.5 \\
& $15 \%$ & 20.0 & 4.1 \\
Swift-BAT & $20 \%$ & 35.2 & 5.6 \\
AGN & $10 \%$ & 39.6 & 6.0 \\
& $15 \%$ & 82.4 & 8.8 \\
& $20 \%$ & 139.3 & 11.6 \\
\hline
\end{tabular}

Table 2: TS and $\sigma$ values for select scenarios with $\Theta^{*}=15^{\circ}$ and $N_{\mathrm{ev}}=1400$.

sets are weighted by the projected exposure over the sky for either POEMMA or ZAP (see Figure 1).

For POEMMA, which has a relatively mature design concept and excellent energy resolution in precision Stereo mode, we perform the first type of statistical analysis for a given energy threshold corresponding to a predetermined number or events. Motivated by the cross-correlation results of Auger, we adopt the threshold energy value of $\sim 40 \mathrm{EeV}$, which corresponds to $N_{\mathrm{ev}} \sim 1400$ events in five years of POEMMA, assuming the Auger cosmic ray spectrum [27]. For each mock data set, we perform the likelihood analysis outlined in Section 2, computing the TS value as a function of the search parameters $f_{\text {sig }}$ and $\Theta$. Since TS values vary for each realization, we simulate 1000 data sets for each scenario and compute the average TS value at particular values of $f_{\text {sig }}$ and $\Theta$ in order to construct the TS profiles (see example in Figure 3). Average TS values and corresponding significances are provided in Table 2 for select astrophysical scenarios with $\Theta^{*}$ (motivated by the anisotropy results of [17]). The results show that POEMMA will be able to detect the crosscorrelation at the level of $5 \sigma$ in many plausible astrophysical scenarios, with the starburst and AGN scenarios being the most promising. However, even in scenarios for the 2MRS catalog, which has a much higher source density than in the other catalogs, there are some parameter regimes in which POEMMA will be able to detect a cross correlation.

For ZAP, for which the instrumental design and requirements are still subject to change, we perform the second type of statistical analysis, allowing the number of events to vary in order to determine the number required in order to guarantee ( $\geq 95 \%$ chance) a $5 \sigma$ detection in certain astrophysical scenarios. For each selection of the parameters $N_{\mathrm{ev}}, f_{\mathrm{an}}^{*}$, and $\Theta^{*}$, we simulate 100 data sets and perform the likelihood test on each one to form a distribution of maximum TS values. We also simulate 100 data sets of $N_{\text {ev }}$ events drawn from isotropy (weighted by the projected exposure over the sky, which we assume to be similar to POEMMA's; see Figure 1) and similarly form a distribution of maximum TS values from the likelihood analysis. For each realization of the parameters $f_{\text {an }}^{*}$ and $\Theta^{*}$, we allow $N_{\text {ev }}$ to vary until the $5^{\text {th }}$-percentile TS value for the anisotropic data sets is more than five standard devations away from the mean of the TS distribution for the 


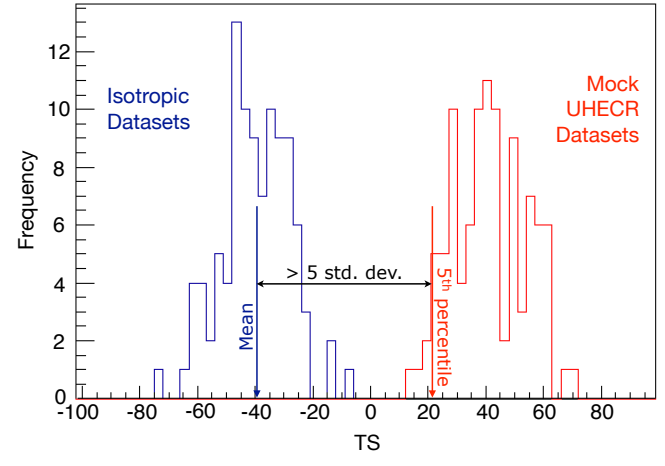

Figure 4: Example TS distributions of purely isotropic (blue) and mock UHECR (red) datasets. The scenario is the same as in Fig. 3 except with $N_{\mathrm{ev}}=2000$.

\begin{tabular}{|c|c|c|c|c|}
\hline \hline \multicolumn{2}{|c|}{ Parameter } & \multicolumn{3}{c|}{$N_{\text {ev }}$ Required } \\
\hline$f_{\text {sig }}$ & $\Theta$ & AGN & SBG & 2 MRS \\
\hline \multirow{2}{*}{$10 \%$} & $20^{\circ}$ & 1240 & 2060 & $>5000$ \\
& $15^{\circ}$ & 920 & 1910 & 4830 \\
\hline \multirow{2}{*}{$15 \%$} & $20^{\circ}$ & 680 & 1000 & 2550 \\
& $15^{\circ}$ & 660 & 870 & 2280 \\
\hline \multirow{2}{*}{$20 \%$} & $20^{\circ}$ & $<650$ & $<650$ & 1520 \\
& $15^{\circ}$ & $<650$ & $<650$ & 1320 \\
\hline
\end{tabular}

Table 3: Required number of events for $\gtrsim 95 \%$ chance of $5 \sigma$ detection for select scenarios with $\Theta^{*}=15^{\circ}$ and $\Theta^{*}=20^{\circ}$.

isotropic data sets (see Figure 4). For this analysis, $f_{\text {an }}^{*}$ and $\Theta^{*}$ are allowed to vary within the bounds of uncertainties in the astrophysical scenario and expectations for ZAP's energy and angular resolutions. Table 3 provides the required number of events for select astrophysical scenarios. Within a mission timescale of two years, ZAP will have the exposure to detect $\gtrsim 2000$ events with energies above $40 \mathrm{EeV}$; as such, ZAP will reach discovery potential in several plausible astrophysical scenarios.

Note that even though the range of parameters in the second type of statistical analysis is motivated by expectations for ZAP's design, the analysis does not explicitly depend on the instrument performance. Furthermore, since we used POEMMA's exposure, the results in Table 3 are also applicable to POEMMA and any other instrument with similar exposure over the sky.

\section{Comparison of Search Parameters and Significances Obtained by Different Experiments}

It is worth noting that while some of the scenarios included in this study are very similar to the maximum-likelihood search parameters obtained by the Auger collaboration [17], the maximum TS values obtained in Table 2 may be somewhat different from the values obtained by Auger. This is due to the fact that the experiments under consideration here will have full-sky coverage, and their sky maps contain sources that are not observable by Auger. The impact is that in simulations in which we assume the same anisotropic fraction as found by Auger, the anisotropic events are now distributed over more sources and spread out over wider portions of the sky, making each individual source less significant.

It is also worth noting that different experiments with different detector performances may obtain different values for the parameters $N_{\mathrm{ev}}, f_{\mathrm{an}}^{*}$, and $\Theta^{*}$. For instance, a higher energy threshold limits the test sample to events that would be located closer to astrophysical sources, resulting in a higher value for $f_{\mathrm{sig}}$, but fewer statistics for the test, lowering the significance of detected cross correlation. A lower energy threshold provides more statistics, but results in a lower value for $f_{\text {sig }}$ by expanding the test sample to events that are less likely to coincide with astrophysical sources. An experiment's energy resolution also plays a role in its measured value of $f_{\text {sig }}$ as uncertainties in energy measurements will result in some events that should be included in the test sample being 
excluded due to spurious lower energy measurements and some events that should be excluded being included due to spurious higher energy measurements. Finally, while the value of $\Theta$ is related to the expected amount of deflection by galactic and extragalactic magnetic fields, an experiment's angular resolution will all contribute its measured value.

Acknowledgements - This work is supported by NASA grants 80NSSC19K0626 at the University of Maryland, Baltimore County, 80NSSC19K0460 at the Colorado School of Mines, 80NSSC19K0484 at the University of Iowa, and 80NSSC19K0485 at the University of Utah, 80NSSC18K0464 at Lehman College, and under proposal 17-APRA17-0066 at NASA/GSFC and JPL. The conceptual design of POEMMA was supported by NASA Probe Mission Concept Study grant NNX17AJ82G for the 2020 Decadal Survey Planning. Contributors to this work were supported in part by NASA awards 16-APROBES16-0023, NNX17AJ82G, NNX13AH54G, 80NSSC18K0246, and 80NSSC18K0473. Research on ZAP was carried out at the Jet Propulsion Laboratory, California Institute of Technology, under a contract with NASA (80NM0018D0004). (C2021 California Institute of Technology. All rights reserved. Work on ZAP has received financial support from Xunta de Galicia (Centro singular de investigación de Galicia accreditation 2019-2022), by European Union ERDF, by the "María de Maeztu" Units of Excellence program MDM-2016-0692, the Spanish Research State Agency and from Ministerio de Ciencia e Innovación PID2019-105544GB-I00.

\section{References}

[1] P. Auger, P. Ehrenfest, R. Maze, J. Daudin, and R. A. Fréon, Rev. Mod. Phys. 11, 288 (1939).

[2] K. Greisen, Phys. Rev. Lett. 16, 748 (1966).

[3] G. T. Zatsepin and V. A. Kuzmin, JETP Lett. 4, 78 (1966), [Pisma Zh. Eksp. Teor. Fiz.4,114(1966)].

[4] D. Allard, Astropart. Phys. 39, 33 (2012), 1111. 3290.

[5] A. Aab et al. (Pierre Auger), Science 357, 1266 (2017), 1709. 07321.

[6] A. Aab et al. (Pierre Auger), Astrophys. J. 891, 142 (2020), 2002 . 06172.

[7] J. Biteau et al., in 37th International Cosmic Ray Conference (these proceedings) (2021).

[8] J. Kim, D. Ivanov, K. Kawata, H. Sagawa, G. Thomson, et al., in 37th International Cosmic Ray Conference (these proceedings) (2021).

[9] J. F. Soriano, L. A. Anchordoqui, D. Torres, et al., in 36th International Cosmic Ray Conference (2019).

[10] J. Biteau et al. (Pierre Auger, Telescope Array), EPJ Web Conf. 210, 01005 (2019), 1905.04188.

[11] F. Sarazin et al., Bull. Am. Astron. Soc. 51, 93 (2019), 1903. 04063.

[12] A. Olinto et al., Journal of Cosmology and Astroparticle Physics 2021, 007 (2021).

[13] A. Romero-Wolf et al., in 37th International Cosmic Ray Conference (these proceedings) (2021).

[14] L. A. Anchordoqui et al., Phys. Rev. D 101, 023012 (2020), 1907.03694.

[15] G. A. Askaryan, Journal of the Physical Society of Japan Supplement 17, 257 (1962).

[16] G. A. Askar'yan, Soviet Journal of Experimental and Theoretical Physics 21, 658 (1965).

[17] L. Caccianiga et al., in 36th International Cosmic Ray Conference (2019).

[18] W. H. Baumgartner, J. Tueller, C. B. Markwardt, G. K. Skinner, S. Barthelmy, R. F. Mushotzky, P. A. Evans, and N. Gehrels, Astrophys. J. Suppl. 207, 19 (2013), 1212. 3336.

[19] J. P. Huchra et al., Astrophys. J. Suppl. 199, 26 (2012), 1108. 0669.

[20] P. Abreu et al. (Pierre Auger), Astropart. Phys. 34, 314 (2010), 1009. 1855.

[21] R. U. Abbasi et al. (Telescope Array), Astrophys. J. Lett. 867, L27 (2018), 1809. 01573.

[22] L. A. Anchordoqui, Phys. Rept. 801, 1 (2019), 1807.09645.

[23] A. Aab et al. (Pierre Auger), Astrophys. J. Lett. 853, L29 (2018), 1801. 06160.

[24] A. Aab et al. (Pierre Auger), JCAP 04, 038 (2017), [Erratum: JCAP 03, E02 (2018)], 1612.07155.

[25] S. S. Wilks, Annals Math. Statist. 9, 60 (1938).

[26] A. Aab et al. (Pierre Auger), Astrophys. J. 804, 15 (2015), 1411. 6111.

[27] V. Verzi et al., in 36th International Cosmic Ray Conference (2019). 


\section{POEMMA Collaboration}

A. V. Olinto, ${ }^{1}$ J. Krizmanic,${ }^{2,3}$ J. H. Adams ${ }^{4}$ R. Aloisio, ${ }^{5}$ L. A. Anchordoqui, ${ }^{6}$ A. Anzalone, ${ }^{7,8}$ M. Bagheri, ${ }^{9}$ D. Barghini, ${ }^{10}$ M. Battisti, ${ }^{10}$ D. R. Bergman, ${ }^{11}$ M. E. Bertaina, ${ }^{10}$ P. F. Bertone, ${ }^{12}$ F. Bisconti, ${ }^{13}$ M. Bustamante, ${ }^{14}$ F. Cafagna, ${ }^{15}$ R. Caruso, ${ }^{16,8}$ M. Casolino, ${ }^{17,}{ }^{18}$ K. Černý, ${ }^{19}$ M. J. Christl, ${ }^{12}$ A. L. Cummings, ${ }^{5}$ I. De Mitri, ${ }^{5}$ R. Diesing, ${ }^{1}$ R. Engel,,${ }^{20}$ J. Eser, ${ }^{1}$ K. Fang, ${ }^{21}$ F. Fenu, ${ }^{10}$ G. Filippatos, ${ }^{22}$ E. Gazda, ${ }^{9}$ C. Guepin, ${ }^{23}$ A. Haungs, ${ }^{20}$ E. A. Hays, ${ }^{2}$ E. G. Judd, ${ }^{24}$ P. Klimov, ${ }^{25}$ V. Kungel, ${ }^{22}$ E. Kuznetsov, ${ }^{4}$ Š. Mackovjak, ${ }^{26}$ D. Mandát, ${ }^{27}$ L. Marcelli, ${ }^{18}$ J. McEnery, ${ }^{2}$ G. Medina-Tanco, ${ }^{28}$ K.-D. Merenda, ${ }^{22}$ S. S. Meyer, ${ }^{1}$ J. W. Mitchell, ${ }^{2}$ H. Miyamoto, ${ }^{10}$ J. M. Nachtman, ${ }^{29}$ A. Neronov,${ }^{30}$ F. Oikonomou, ${ }^{31}$ Y. Onel, ${ }^{29}$ G. Osteria, ${ }^{32}$ A. N. Otte, ${ }^{9}$ E. Parizot,${ }^{33}$ T. Paul, ${ }^{6}$ M. Pech, ${ }^{27}$ J. S. Perkins, ${ }^{2}$ P. Picozza, ${ }^{18,34}$ L. W. Piotrowski, ${ }^{35}$ Z. Plebaniak, ${ }^{10}$ G. Prévôt,${ }^{33}$ P. Reardon, ${ }^{4}$ M. H. Reno, ${ }^{29}$ M. Ricci, ${ }^{36}$ O. Romero Matamala, ${ }^{9}$ F. Sarazin, ${ }^{22}$ P. Schovánek, ${ }^{27}$ V. Scotti, ${ }^{32,37}$ K. Shinozaki, ${ }^{38}$ J. F. Soriano, ${ }^{6}$ F. Stecker, ${ }^{2}$ Y. Takizawa, ${ }^{17}$ R. Ulrich, ${ }^{20}$ M. Unger, ${ }^{20}$ T. M. Venters, ${ }^{2}$ L. Wiencke, ${ }^{22}$ D. Winn, ${ }^{29}$ R. M. Young, ${ }^{12}$ M. Zotov ${ }^{2}$

${ }^{1}$ The University of Chicago, Chicago, IL, USA ${ }^{2}$ NASA Goddard Space Flight Center, Greenbelt, MD, USA ${ }^{3}$ Center for Space Science \& Technology, University of Maryland, Baltimore County, Baltimore, MD, USA ${ }^{4}$ University of Alabama in Huntsville, Huntsville, AL, USA ${ }^{5}$ Gran Sasso Science Institute, L'Aquila, Italy ${ }^{6}$ City University of New York, Lehman College, NY, USA ${ }^{7}$ Istituto Nazionale di Astrofisica INAF-IASF, Palermo, Italy ${ }^{8}$ Istituto Nazionale di Fisica Nucleare, Catania, Italy ${ }^{9}$ Georgia Institute of Technology, Atlanta, GA, USA ${ }^{10}$ Universita' di Torino, Torino, Italy ${ }^{11}$ University of Utah, Salt Lake City, Utah, USA ${ }^{12}$ NASA Marshall Space Flight Center, Huntsville, AL, USA ${ }^{13}$ Istituto Nazionale di Fisica Nucleare, Turin, Italy ${ }^{14}$ Niels Bohr Institute, University of Copenhagen, DK-2100 Copenhagen, Denmark ${ }^{15}$ Istituto Nazionale di Fisica Nucleare, Bari, Italy ${ }^{16}$ Universita' di Catania, Catania Italy ${ }^{17}$ RIKEN, Wako, Japan ${ }^{18}$ Istituto Nazionale di Fisica Nucleare, Section of Roma Tor Vergata, Italy ${ }^{19}$ Joint Laboratory of Optics, Faculty of Science, Palacký University, Olomouc, Czech Republic ${ }^{20}$ Karlsruhe Institute of Technology, Karlsruhe, Germany ${ }^{21}$ Kavli Institute for Particle Astrophysics and Cosmology, Stanford University, Stanford, CA94305, USA ${ }^{22}$ Colorado School of Mines, Golden, CO, USA ${ }^{23}$ Department of Astronomy, University of Maryland, College Park, MD, USA ${ }^{24}$ Space Sciences Laboratory, University of California, Berkeley, CA, USA ${ }^{25}$ Skobeltsyn Institute of Nuclear Physics, Lomonosov Moscow State University, Moscow,Russia ${ }^{26}$ Institute of Experimental Physics, Slovak Academy of Sciences, Kosice, Slovakia ${ }^{27}$ Institute of Physics of the Czech Academy of Sciences, Prague, Czech Republic ${ }^{28}$ Instituto de Ciencias Nucleares, UNAM, CDMX, Mexico ${ }^{29}$ University of Iowa, Iowa City, IA, USA ${ }^{30}$ University of Geneva, Geneva, Switzerland ${ }^{31}$ Institutt for fysikk, NTNU, Trondheim, Norway ${ }^{32}$ Istituto Nazionale di Fisica Nucleare, Napoli, Italy ${ }^{33}$ Université de Paris, CNRS, Astroparticule et Cosmologie, F-75013 Paris, France ${ }^{34}$ Universita di Roma Tor Vergata, Italy ${ }^{35}$ Faculty of Physics, University of Warsaw, Warsaw, Poland ${ }^{36}$ Istituto Nazionale di Fisica Nucleare - Laboratori Nazionali di Frascati, Frascati, Italy ${ }^{37}$ Universita' di Napoli Federico II, Napoli, Italy ${ }^{38}$ National Centre for Nuclear Research, Lodz, Poland

\section{ZAP Collaboration}

A. Romero-Wolf, ${ }^{1}$ J. Alvarez-Muñiz, ${ }^{2}$ L. A. Anchordoqui, ${ }^{3}$ D. Bergman, ${ }^{4}$ W. Carvalho, Jr., ${ }^{5}$ A. L. Cummings, ${ }^{6}$ P. Gorham, ${ }^{7}$ C. J. Handmer, ${ }^{1}$ N. Harvey, ${ }^{1}$ J. F. Krizmanic, 8,9 K. Nishimura, ${ }^{7}$ M. H. Reno, ${ }^{1} 0$ H. Schoorlemmer, ${ }^{1} 1$ G. Varner, ${ }^{7}$ T. M. Venters, ${ }^{8}$ S. Wissel, ${ }^{1} 2$ E. Zas ${ }^{2}$

${ }^{1}$ Jet Propulsion Laboratory, California Institute of Technology, Pasadena, CA, USA ${ }^{2}$ IGFAE \& Universidade Santiago de Compostela, Spain ${ }^{3}$ Lehman College, City University of New York, New York, NY, USA ${ }^{4}$ University of Utah, Salt Lake City, UT, USA ${ }^{5}$ Universidade de São Paulo, Brazi ${ }^{6}$ Gran Sasso Science Institute, Italy ${ }^{7}$ University of Hawai'i at Mānoa, Honolulu, HI, USA ${ }^{8}$ NASA Goddard Space Flight Center, Greenbelt, MD, USA ${ }^{9}$ Center for Space Science \& Technology, University of Maryland, Baltimore County, Baltimore, MD, USA ${ }^{1}$ OUniversity of Iowa, Iowa City, IA, USA ${ }^{1} 1$ Max Planck Institute für Kernphysik, Germany ${ }^{1} 2$ Pennsylvania State University, State College, PA, USA 